\title{
Multi-wavelength study of the gravitational lens system RXS J113155.4-123155
}

\section{Multi-epoch optical and near infrared imaging ${ }^{\star}$}

\author{
D. Sluse ${ }^{1,2,3}$, J.-F. Claeskens ${ }^{1}$, B. Altieri ${ }^{4}$, R. A. Cabanac ${ }^{5}$, O. Garcet ${ }^{1}$, D. Hutsemékers ${ }^{1, \star \star}$, C. Jean ${ }^{1}$, \\ A. Smette $\mathrm{e}^{1,2, \star \star}$, and J. Surdej ${ }^{1, \star \star \star}$
}

\author{
${ }^{1}$ Institut d'Astrophysique et de Géophysique, Université de Liège, Allée du 6 Août 17, B5C, 4000 Sart Tilman, Belgium \\ e-mail: dominique.sluse@epfl.ch \\ 2 European Southern Observatory, Alonso de Cordova 3107, Santiago 19, Chile \\ ${ }^{3}$ Laboratoire d'Astrophysique, École Polytechnique Fédérale de Lausanne (EPFL) Observatoire, 1290 Sauverny, Switzerland \\ 4 European Space Astronomy Centre, ESA, PO Box 50727, 28080 Madrid, Spain \\ 5 Canada-France-Hawaii Telescope, 65-1238 Mamalahoa Highway, Kamuela, HI 96743, USA
}

Received 29 March 2005 / Accepted 28 November 2005

\section{ABSTRACT}

Aims. RXS J113155.4-123155 $(z=0.66)$ is a quadruply imaged lensed quasar with a resolved Einstein Ring. The goal of this paper is to provide a full characterization of this system, and more particularly accurate astrometry and photometry. These observational constraints constitute a mandatory ingredient for the precise determination of the lens mass profile, the derivation of the Hubble constant $H_{0}$ from time delay measurements and investigations on the presence of massive substructures in the lensing galaxy.

Methods. Visible and near-infrared imaging observations of RXS J113155.4-123155 were carried out at various epochs using several ground based telescopes and the HST. The frames have been deconvolved using the MCS algorithm. A Singular Isothermal Ellipsoid (SIE) + external shear has been used to model the lensing galaxy potential.

Results. MCS deconvolution enables us to separate the flux of the QSO (point-like images) from that of its host galaxy and to accurately track the flux variations of the point-like images in various filters. The deconvolved frames unveil several multiply imaged structures in the Einstein ring and an unidentified object in the vicinity of the lensing galaxy. We discuss the lightcurves and the chromatic flux ratio variations and deduce that both intrinsic variability and microlensing took place during a span longer than one year.

We demonstrate that microlensing may easily account for the so called anomalous flux ratios presented in the discovery paper. However, the observed flux ratios are still poorly reproduced when modeling the lens potential with a SIE+shear. We argue that this disagreement can hardly be explained by milli-lensing caused by substructures in the lensing galaxy. A solution proposed in Paper II consists in a more complex lens model including an octupole term to the lens gravitational potential.

Key words. gravitational lensing - galaxies: Seyfert - quasars: individual: RXS J113155.4-123155

\footnotetext{
* Based on observations collected at the European Southern Observatory, La Silla and Paranal, Chile (ESO Program 71.A-0407(A, E) and DDT Program 272.A-0535(A)); on observations obtained with the NASA/ESA Hubble Space Telescope (Space Telescope Science Institute) which is operated by the Association of Universities for Research in Astronomy, Inc., under NASA contract NAS 5-26555 (program \#9744); and on observations obtained at the Canada-FranceHawaii Telescope (CFHT) which is operated by the National Research Council of Canada, the Institut National des Sciences de l'Univers of the Centre National de la Recherche Scientifique of France, and the University of Hawaii.

$\star \star$ Chercheur qualifié du FNRS (Belgique).

$\star \star \star$ Directeur de recherches honoraire du FNRS (Belgique).
}

\section{Introduction}

RXS J113155.4-123155 (hereafter J1131) is one of the nearest confirmed multiply imaged AGN. The source at $z_{\mathrm{s}}=0.658$ is lensed by an elliptical galaxy at $z_{1}=0.295$ (Sluse et al. 2003, hereafter Paper I). This system is a long axis quad with an image configuration very similar to B1422+231 (Patnaik et al. 1992): three merging images (B-A-C; typical of a source lying close to a cusp caustic) face the faint saddle-point image D lying close to the lensing galaxy $(\mathrm{G})$. For such a system (i.e. cusp configuration system), the magnification behaviour is well understood - the flux of the middle image should be equal to the total fluxes of the two outer images (Schneider \& Weiss 1992). 
However, this generic prediction was strongly violated at the epoch of the discovery, suggesting the likely presence of smallscale structures in the lensing galaxy (Keeton et al. 2003, 2005; hereafter KGP03, KGP05). Thanks to the data set presented here, we re-examine the values of the flux-ratios with regard to their temporal and chromatic variations. Additionally, because this quad is quite bright and shows lensed images with a wide angular separation $\left(2 \theta_{\mathrm{E}} \sim 3.6^{\prime \prime}\right)$, it is potentially a target of interest for time delay measurements. Also, the bright Einstein ring detected from the optical to the near-infrared offers unique constraints on the lens modeling (Kochanek et al. 2001) that may break the degeneracy between the intrinsic lens galaxy shape and the external shear (Keeton et al. 2000). However, the bright Einstein ring introduces an additional complexity to the photometry measurement because it is superimposed over the lensed point-like images. This motivates our extensive discussion of the sources of systematic photometric errors affecting the individual lensed QSO images and of the photometric accuracy that can be reached for $\mathrm{J} 1131$.

The observations presented here constitute the first part of a follow-up study of this system that also includes detailed lens models and source reconstruction from HST imaging, X-ray imaging as well as optical and NIR spectroscopy. Sections 2 and 3 describe the data reduction and analysis. Section 4 includes a description of the ring morphology, a simple modeling of the lensing galaxy and a discussion of the flux (time and chromatic) variability of the lensed images. Finally, Sect. 5 summarizes the main results and presents our conclusions.

We adopt throughout this paper $H_{0}=65 \mathrm{~km} \mathrm{~s}^{-1} \mathrm{Mpc}^{-1}$, $\Omega_{0}=0.3$ and $\Lambda_{0}=0.7$; magnitudes are computed in the Vega system.

\section{Observations and reductions}

In this section, we present NIR and optical imaging obtained for J1131. Near Infrared observations include images recorded with ESO telescopes, with CFHT and HST. The images recorded at visible wavelengths have been obtained with the FORS1 and FORS2 instruments at the Cassegrain focus of Antu (UT1) and Yepun (UT4) in Paranal. Table 1 summarizes the technical data of the various instrumentations used and lists the observational characteristics of each data set.

\subsection{Ground-based NIR observations}

First, $J$ band images of J1131 have been obtained with SOFI. The conditions were photometric and 6 standard stars have been observed during the night enabling us to derive the magnitude of an object producing 1 count/s of $23.15 \pm 0.01$ (hereafter zero-point).

Secondly, images of J1131 have been obtained in the Ksband with the Short Wavelength Imaging camera of the ISAAC instrument. Thanks to the short DIT (10s) used, the combined flux from the sky and QSO images in any pixel never exceeded 10000 ADU, a level below the non-linearity regime of the ISAAC detector. Conditions were photometric and a zero-point of $24.17 \pm 0.07$ has been deduced from the standard stars observed during the night.
Finally, a set of NIR images of J1131 has been obtained at a single epoch with CFHT-IR through the broad band J\#5133, H\#5209 and $K^{\prime} \# 5337$ filters.

For the whole set of data, standard NIR reduction procedures were applied to subtract the dark and to flat field the images using a normalized flat field. Sky subtraction and coaddition of the reduced dithered frames were performed using the xdimsum IRAF $^{1}$ package. The residual sky pattern has been fitted and subtracted using the SExtractor software v 2.3.2 (Bertin 1996).

\subsection{Ground-based optical observations}

Observations with the FORS2 instrument have been obtained with the High Resolution (HR) collimator and the CCD detector in the $2 \times 2$ binning mode. Conditions were not photometric.

$B, V, R, I$ images of $\mathrm{J} 1131$ were obtained in April 2004 under photometric conditions with the FORS1 instrument and a CCD in the unbinned mode. Zero-points for the Bessel $B$, $V, R, I$ filters were found to be $27.76 \pm 0.02,28.12 \pm 0.02$, $28.06 \pm 0.04$ and $27.25 \pm 0.01$ respectively.

Standard reduction procedures were applied to the data including bias subtraction and flat fielding with a normalized flat. The photometric measurements of the standard stars have been performed with the phot IRAF task. The sky has been subtracted using the SExtractor software v 2.3.2 (Bertin 1996).

\subsection{HST F160W observations}

HST F160W observations of J1131 have been obtained on November 17, 2003 with the Hubble Space Telescope (HSTGO-9744; PI: C.S. Kochanek) as part of the CfA-Arizona Space Telescope Lens Survey (CASTLES). Out of the eight dithered frames of J1131 obtained with the NIC2, two frames have been excluded from our analysis because of an error flag associated with pixels located in the brightest point-like images. We reduced the images using the IRAF calnica and calnicb tasks as recommended in the NICMOS data handbook (Dickinson et al. 2002). The flux calibration (Dickinson et al. 2002) has been derived from PHOTNU $=1.4981 \times$ $10^{-6} \mathrm{Jy} \mathrm{s} \mathrm{DN}^{-1}$ and from the averaged flux density of VEGA $\left\langle F_{v}\right\rangle=1043.5 \mathrm{Jy}$ (June 2004 values).

\section{Data analysis}

\subsection{The method}

The Einstein ring of J1131 is the brightest one known today. Because it is superimposed over the lensed QSO images, standard photometric methods like PSF fitting may bias the point-like flux measurements. We thus measured relative photometry using the MCS deconvolution method (Magain et al. 1998) which proved to be very efficient in measuring fluxes

\footnotetext{
${ }^{1}$ IRAF is distributed by the National Optical Astronomy Observatories, which are operated by the Association of Universities for Research in Astronomy, Inc., under cooperative agreement with the National Science Foundation.
} 
Table 1. Log of the observations. Epoch $=$ epoch number as used in the text. CONAD = electron to ADU conversion factor. NExp $=$ number of dithered frames. Exp $=$ total integration time of a single frame. For the NIR data, Exp $=$ NDIT $\times$ DIT, where NDIT is the number of subframes with an integration time of DIT seconds. Only the total exposure time per frame is reported for the NIC 2 images. $F W H M=$ mean seeing measured on frames. Note that the SOFI pixel size has been re-measured to be $0.289^{\prime \prime}$, superseding the published ESO value of $0.292^{\prime \prime}$.

\begin{tabular}{cccccccccc}
\hline \hline $\begin{array}{c}\text { Date } \\
(\text { dd-mm-yyyy) }\end{array}$ & Epoch & $\begin{array}{c}\text { Instrument } \\
(\text { Telescope) }\end{array}$ & $\begin{array}{c}\text { RON } \\
\left(\mathrm{e}^{-} / \text {pixel) }\right.\end{array}$ & $\begin{array}{c}\text { CONAD } \\
\left(\mathrm{e}^{-} / \text {ADU) }\right.\end{array}$ & $\begin{array}{c}\text { Pix. size } \\
\left({ }^{\prime \prime}\right)\end{array}$ & Filter & NExp & $\begin{array}{c}\text { Exp } \\
(\mathrm{s})\end{array}$ & $\begin{array}{c}F W H M \\
\left({ }^{\prime \prime}\right)\end{array}$ \\
\hline $21-11-2002$ & 1 & SOFI (NTT) & 11 & 5.3 & 0.289 & $J$ & 24 & $2 \times 30$ & $0.7-0.9$ \\
$12-04-2003$ & 2 & ISAAC (UT1) & 11 & 4.6 & 0.1484 & $K$ s & 48 & $6 \times 10$ & $0.3-0.55$ \\
$21-04-2003$ & 3 & FORS2 (UT4) & 4.2 & 1.25 & 0.1263 & $R$ special & 4 & 80 & $0.65-0.85$ \\
$02-05-2003$ & 4 & FORS2 (UT4) & 4.2 & 1.25 & 0.1263 & $R$ special & 6 & 40 & 0.8 \\
$26-05-2003$ & 5 & FORS2 (UT4) & 4.2 & 1.25 & 0.1263 & $B$ Bessel & 8 & 497 & $0.65-0.9$ \\
$26-05-2003$ & 5 & FORS2 (UT4) & 4.2 & 1.25 & 0.1263 & $B$ Bessel & 7 & 125 & $0.75-1.0$ \\
$17-06-2003$ & 6 & FORS2 (UT4) & 4.2 & 1.25 & 0.1263 & $R$ special & 6 & 40 & $0.45-0.50$ \\
$17-06-2003$ & 6 & FORS2 (UT4) & 4.2 & 1.25 & 0.1263 & $V$ Bessel & 20 & 59 & $0.5-0.65$ \\
$17-11-2003$ & 7 & NIC2 (HST) & 26 & 5.4 & 0.075 & $F 160 W$ & 5 & 640 & 0.12 \\
$17-11-2003$ & 7 & NIC2 (HST) & 26 & 5.4 & 0.075 & $F 160 W$ & 3 & 704 & 0.12 \\
$18-11-2003$ & 7 & FORS2 (UT4) & 4.2 & 1.25 & 0.1263 & $B$ Bessel & 3 & 240 & 1.0 \\
$18-11-2003$ & 7 & FORS2 (UT4) & 4.2 & 1.25 & 0.1263 & $V$ Bessel & 4 & 120 & $0.8-1.0$ \\
$18-11-2003$ & 7 & FORS2 (UT4) & 4.2 & 1.25 & 0.1263 & $R$ special & 4 & 80 & $0.85-0.95$ \\
$09-02-2004$ & 8 & CFHT-IR (CFHT) & 15 & 2.35 & 0.211 & $J \# 5133$ & 4 & $6 \times 120$ & $0.6-0.8$ \\
$09-02-2004$ & 8 & CFHT-IR (CFHT) & 15 & 2.35 & 0.211 & $H \# 5209$ & 4 & $6 \times 60$ & $0.6-0.8$ \\
$09-02-2004$ & 8 & CFHT-IR (CFHT) & 15 & 2.35 & 0.211 & $K^{\prime} \# 5337$ & 8 & $3 \times 60$ & $0.55-0.9$ \\
$12-04-2004$ & 9 & FORS1 (UT1) & 5.2 & 1.61 & 0.100 & $B$ Bessel & 3 & 60 & $0.7-0.8$ \\
$12-04-2004$ & 9 & FORS1 (UT1) & 5.2 & 1.61 & 0.100 & $V$ Bessel & 4 & 30 & $0.65-0.85$ \\
$12-04-2004$ & 9 & FORS1 (UT1) & 5.2 & 1.61 & 0.100 & $R$ Bessel & 4 & 20 & $0.7-0.85$ \\
$12-04-2004$ & 9 & FORS1 (UT1) & 5.2 & 1.61 & 0.100 & $I$ Bessel & 4 & 20 & $0.6-0.7$ \\
\hline
\end{tabular}

of point-like components superimposed over a varying background (Burud et al. 1998). Thanks to this code, images are deconvolved to an improved but finite resolution using a kernel constructed from PSF stars (Courbin et al. 1998). For HST data, the kernel is constructed based on the PSF generated with the Tiny Tim v 6.1 software (Krist \& Hook 2003). In the other cases, the kernel is constructed from relatively isolated bright field stars. Additionally, since the co-addition of different images often degrades the PSF, we generally worked on the individual frames obtained at different dithered positions.

Instead of deconvolving each image separately, we used a modified version of the code that simultaneously deconvolves a set of individual frames taken with the same instrumental setup (Burud et al. 2000), leading to better constraints on the astrometry of the quasar images (an output of the deconvolution process), on the shape of the Einstein ring and of the lensing galaxy, using the $\mathrm{S} / \mathrm{N}$ of the whole data set. For each data set, deconvolved frames are reconstructed to a common improved resolution. Technically, the relative positions of the point-like components are fitted on the reference frame while their flux and the frame offset with respect to the reference vary independently for each frame. For the data sets with the largest pixel size (i.e. CFHT-IR and SOFI), MCS could not fit accurately both the offset with respect to the reference frame and the point-like image positions. In that case, the latter have been fixed to the ones derived from the HST observations.

In the following, we have simultaneously deconvolved all the frames: i) for each epoch (in a given filter) separately; ii) for all the epochs available (in a filter). Single epoch deconvolution is best suited to retrieve absolute photometry (Sect. 3.3.1) since it is not affected by the flux scaling between several epochs.
It is also the only method applicable to the $I$ band and NIR filters (i.e. one epoch data). Multi-epoch deconvolution is more appropriate to retrieve accurate flux ratios (Sect. 3.3.2) and to measure the temporal flux variations (Sects. 3.3.2 and 4.3). Typical photometric random errors obtained with MCS reach the photon noise uncertainty.

Although the MCS deconvolution enables one to retrieve accurate photometry, the results are affected by systematic errors (Burud et al. 1998) mainly associated with the regularization term $\lambda$. The latter works like a local smoothing term by reducing the high frequencies of the background. Thus, depending on the choice ${ }^{2}$ of $\lambda$, a variable fraction of the background flux is included in the point-like flux measurements. We selected realistic values of $\lambda$ based on the flatness of the residual map (i.e. the difference between the real image and the deconvolved image re-convolved with the kernel, and divided by the standard deviation in each pixel of the real image). More specifically, the higher level of regularization is picked when we observe more than $3 \sigma$ standard deviation in the residual map (regularization is swamping -true- structures existing in the original image) and the lower level is chosen when important Gibbs oscillations appear in the deconvolved frame. In the following, we run MCS with three acceptable levels of regularization: a lower and upper bound - resp. $\lambda_{\text {low }}$ and $\lambda_{\text {high }}-$ and an intermediate value $\lambda_{\text {best }}$ considered as the best value.

Another source of systematic photometric errors may come from an imperfect PSF model. However, this appears as deviations from a null residual map and the associated photometric

\footnotetext{
${ }^{2}$ A high value of $\lambda$ means a small regularization and a low value means a strong smoothing of the deconvolved image.
} 
bias is corrected by means of the comparison of deconvolution and aperture photometry of field stars (see e.g. Sect. 3.3.1). Note that in such cases, since the residual map cannot be used to pick up $\lambda_{\text {low }}$ and $\lambda_{\text {high }}$, we select them based on the visual inspection of the deconvolved frame: for a too small value of $\lambda$, the background does not look like a ring+lens galaxy, but just like a very diffuse background while we determine $\lambda_{\text {high }}$ via the appearance of important Gibbs oscillations.

By default, the error bars reported in this paper are $1 \sigma$ standard errors on the mean.

\subsection{Astrometry}

The relative astrometry is derived from the HST data using two methods. First, we performed 2D gaussian fitting of the individual lensed images and of the lensing galaxy (each component isolated in a box of $13 \times 13$ pixels) with the i2gaussfit IRAF task. Mean positions and associated errors are calculated from the measurements on the 6 individual frames.

In the second method, the relative astrometry of images B, $\mathrm{C}, \mathrm{D}$ and of the lens galactic nucleus $\mathrm{G}$ relative to $\mathrm{A}$ is derived simultaneously using MCS. Unfortunately, this method suffers from the poor PSF sampling and is very sensitive to the prior knowledge of the relative offset between the frames. Hence, we have used the gaussian fitting results to fix the mean relative offset and measured the mean position of object $X$ (see Sect. 4.1) with MCS. Note that MCS positions of A, B, C, D and $\mathrm{G}$ are in statistical agreement with the gaussian fitting results.

The measured positions have been converted to the RA/Dec system using a plate scale $^{3}$ of $0.07588^{\prime \prime} /$ pixel in $x$ and of $0.07537^{\prime \prime} /$ pixel in $y$ and a rotation angle of $59.202^{\circ}(\mathrm{E}$ of $\mathrm{N})$.

Relative astrometry is also derived from the best groundbased observations (i.e. FORS2 and ISAAC data). Since the spatial sampling of the ground-based PSFs fully satisfies the sampling theorem, MCS leads to an accurate relative astrometry. The relative positions of the lensed quasar images and of the lensing galaxy found in each band (namely $B, V, R$ and $K \mathrm{~s}$ ) do agree between each other, within typically less than $0.01^{\prime \prime}$. The standard deviation of the positions measured in each filter is used to derive the $1 \sigma$ standard error on the mean relative positions. However, because of the small separation of the triplet of images A, B and C, the measured positions of B and C are likely correlated with that of $\mathrm{A}$, resulting in an underestimate of the error. Consequently, we have arbitrarily and quadratically added a $0.001^{\prime \prime}$ error to the $1 \sigma$ standard errors of B and $\mathrm{C}$.

The systematic error on the position is estimated to be $0.003^{\prime \prime}$. This error is typical of the systematic error that may affect the relative astrometry of small separation systems on NIC2 data (Impey et al. 1998). For the ground-based data, the systematic error is inferred from the recalibration of the pixel size of the FORS frames with the MIDAS implementation of

\footnotetext{
3 http://WwW.stsci.edu/hst/nicmos/performance/ platescale
}

Table 2. Relative positions of the different lensed components (BCD), the lensing galaxy $(\mathrm{G})$ and the companion object $(\mathrm{X})$ with respect to $\mathrm{A}$. These are deduced from a gaussian fitting (except $\mathrm{X}$ with MCS) on the HST NIC2 image $(F 160 W)$ and from the MCS deconvolution of the FORS 2 and ISAAC frames. The last column lists the $1 \sigma$ random error in both right ascension and declination. For the ground-based data, the error on the relative position is reported. $1 \sigma$ systematic errors related to scaling, orientation and distortion uncertainties are estimated to be $0.003^{\prime \prime}$ (see text for details).

\begin{tabular}{c|rr|rr|cc}
\hline \hline ID & \multicolumn{2}{|c}{$\Delta \alpha \cos \delta\left(^{\prime \prime}\right)$} & \multicolumn{2}{c}{$\Delta \delta\left({ }^{\prime \prime}\right)$} & \multicolumn{2}{c}{$1 \sigma\left(^{\prime \prime}\right)$} \\
\hline & $F 160 W$ & ground & $F 160 W$ & ground & $F 160 W$ & ground \\
\hline A & 0 & 0 & 0 & 0 & 0.001 & - \\
$\mathrm{B}$ & 0.032 & 0.030 & 1.184 & 1.190 & 0.001 & 0.001 \\
$\mathrm{C}$ & -0.590 & -0.589 & -1.117 & -1.117 & 0.001 & 0.002 \\
$\mathrm{D}$ & -3.115 & -3.120 & 0.875 & 0.881 & 0.001 & 0.002 \\
$\mathrm{G}$ & -2.027 & -2.027 & 0.607 & 0.604 & 0.001 & 0.002 \\
$\mathrm{X}$ & -1.936 & - & 1.100 & - & 0.011 & - \\
\hline
\end{tabular}

pos1 (Walter \& West 1986) and with $\mathrm{GAIA}^{4}$ (v 2.5.3). Table 2 summarizes the relative positions we derived for J1131 based on the HST and ground-based data. Both sets of results do agree within $0.006^{\prime \prime}$.

\subsection{Photometry}

\subsubsection{Absolute photometry}

We derive absolute photometric measurements with MCS in the Bessel $B, V, R, I$ bands and for the $J, F 160 W$ and $K$ s filters (Table 3 ) based on the fluxes obtained with $\lambda_{\text {best }}$. Sets of individual frames are used except for the $J$-SOFI $(K \mathrm{~s})$ filters where a set of $4(8)$ stacked frames of $6 \times 1$ minutes is considered. The peak to peak systematic variations introduced by $\lambda$ are estimated from the results obtained for $\lambda_{\text {low }}$ and $\lambda_{\text {high }}$. Fluxes are converted into the Vega magnitude system using the zero-points reported in Sect. 2.1 and applying an extinction correction (see the ESO webpages for extinction coefficients). Additionally, for each set of ground-based images, we compared the aperture photometry obtained with the phot IRAF routine and SExtractor to MCS photometry of at least 4 stars in the field. All the measurements are consistent, suggesting the absence of systematic errors caused by the estimate of the PSF.

For the HST NIC2 data, the Tiny Tim PSF poorly reproduces the observations. Consequently, we find several acceptable solutions for each possible value of $\lambda\left(\lambda_{\text {best }}\right.$ but also $\left.\lambda_{\text {low,high }}\right)$ suggesting that the minimization routine in MCS is trapped in one of the numerous local minima. Hence, the random error bars of the F160W photometry presented in Table 3 are likely underestimated and the associated peak to peak variations may also be biased. Although we do not know by which amount, we trust these results since they are found in good agreement with independent PSF fitting photometry presented in Claeskens et al. (accepted, hereafter Paper II).

\footnotetext{
${ }^{4}$ GAIA is a derivative of the Skycat catalog and image display tool, developed as part of the VLT project at ESO. Skycat and GAIA are free softwares under the terms of the GNU copyright.
} 
Table 3. Photometry in the $B-V-R-I$ Bessel filters (epoch 9), in the $J$ band (epoch 1), in the $H$-band ( $F 160 W$ Vega normalized; epoch 7) and in the $K \mathrm{~s}$ band (epoch 2) for images A, B, C and D of J1131 as obtained with MCS. The notation is $m_{\text {best }} \pm \sigma_{\text {random }}^{+ \text {sys }}$, where $m_{\text {best }}$ is the mean value obtained for $\lambda_{\text {best }}, \sigma_{\text {random }}$ is the quadratic sum of the $1 \sigma$ error on the mean, the error on the extinction coefficient and the error on the zero-point (this one amounts to $0.03 \mathrm{mag}$ for NICMOS photometry) and \pm sys is the peak to peak variation between extreme values of $\lambda$. Last column gives the FWHM (in arcsec) of the PSF in the deconvolved frame. When null error bars appear in the table, the error bar is of only a few milli-magnitudes.

\begin{tabular}{ccccccc}
\hline \hline Filter & epoch & $\mathrm{A}$ & $\mathrm{B}$ & $\mathrm{C}$ & $\mathrm{D}$ & $F W H M$ \\
\hline$B$ & 9 & $18.09 \pm 0.02_{-0.00}^{+0.00}$ & $18.14 \pm 0.02_{-0.00}^{+0.00}$ & $19.19 \pm 0.02_{-0.01}^{+0.02}$ & $20.64 \pm 0.02_{-0.01}^{+0.03}$ & 0.30 \\
$V$ & 9 & $17.88 \pm 0.02_{-0.01}^{+0.02}$ & $17.83 \pm 0.02_{-0.01}^{+0.03}$ & $18.96 \pm 0.02_{-0.00}^{+0.07}$ & $20.41 \pm 0.02_{-0.06}^{+0.11}$ & 0.30 \\
$R$ & 9 & $17.76 \pm 0.04_{-0.02}^{+0.02}$ & $17.66 \pm 0.04_{-0.03}^{+0.04}$ & $18.77 \pm 0.04_{-0.05}^{+0.10}$ & $20.21 \pm 0.04_{-0.06}^{+0.04}$ & 0.35 \\
$I$ & 9 & $17.43 \pm 0.01_{-0.01}^{+0.05}$ & $17.42 \pm 0.01_{-0.02}^{+0.07}$ & $18.44 \pm 0.01_{-0.02}^{+0.14}$ & $19.72 \pm 0.01_{-0.03}^{+0.03}$ & 0.30 \\
$J$ & 1 & $16.83 \pm 0.03_{-0.01}^{+0.05}$ & $16.49 \pm 0.03_{-0.00}^{+0.03}$ & $17.48 \pm 0.04_{-0.02}^{+0.04}$ & $19.00 \pm 0.04_{-0.01}^{+0.02}$ & 0.29 \\
$F 160 W$ & 7 & $15.80 \pm 0.04_{-0.17}^{+0.04}$ & $16.08 \pm 0.03_{-0.02}^{+0.03}$ & $16.95 \pm 0.04_{-0.04}^{+0.21}$ & $18.36 \pm 0.07_{-0.02}^{+0.03}$ & 0.08 \\
$K \mathrm{~s}$ & 2 & $14.72 \pm 0.07_{-0.00}^{+0.01}$ & $15.11 \pm 0.07_{-0.01}^{+0.00}$ & $15.72 \pm 0.07_{-0.02}^{+0.02}$ & $17.17 \pm 0.07_{-0.03}^{+0.02}$ & 0.15 \\
\hline
\end{tabular}

Table 4. Magnitude differences (Sect. 3.3.2). The notation is $m_{\text {best }} \pm \sigma_{\text {random }}^{+ \text {sys }}$, where $m_{\text {best }}$ is the mean value obtained for $\lambda_{\text {best }}, \sigma_{\text {random }}$ is the $1 \sigma$ error on the mean and \pm sys refers to the peak to peak variations between extreme values of $\lambda$; Col. 1: filter; Col. 2: epoch for which the magnitude differences are given; Col. 3: $R_{i j}(\mathrm{~A})=I_{i} / I_{j}$ for image A. $i$ is the reference epoch (bold face) for the filter considered while $j$ is the epoch given in Col. 2; Cols. 4-6: average magnitude differences between image A and resp. B, C, D; Cols. 7-8: idem between image B and resp. C, D; Col. 9: FWHM (in arcsec) of the PSF in the deconvolved frame. Since the flux variations (Col. 3) are not affected by the regularization, systematic error bars are not expressed. When null error bars appear in the table, the error bar is only of a few milli-magnitudes.

\begin{tabular}{|c|c|c|c|c|c|c|c|c|}
\hline Filter & $\mathrm{E}$ & $R_{i j}(\mathrm{~A})$ & $\Delta m_{\mathrm{AB}}$ & $\Delta m_{\mathrm{AC}}$ & $\Delta m_{\mathrm{AD}}$ & $\Delta m_{\mathrm{BC}}$ & $\Delta m_{\mathrm{BD}}$ & $F W H M$ \\
\hline$B$ & 5 & - & $0.19 \pm 0.00_{-0.00}^{+0.00}$ & $-0.92 \pm 0.00_{-0.00}^{+0.00}$ & $-2.31 \pm 0.00_{-0.01}^{+0.01}$ & $-1.11 \pm 0.00_{-0.00}^{+0.00}$ & $-2.50 \pm 0.00_{-0.01}^{+0.01}$ & 0.30 \\
\hline$B$ & 7 & $0.96 \pm 0.04$ & $-0.01 \pm 0.00_{-0.00}^{+0.00}$ & $-1.06 \pm 0.01_{-0.00}^{+0.00}$ & $-2.36 \pm 0.00_{-0.01}^{+0.00}$ & $-1.05 \pm 0.01_{-0.00}^{+0.00}$ & $-2.35 \pm 0.00_{-0.01}^{+0.01}$ & 0.30 \\
\hline$B$ & 9 & $1.00 \pm 0.04$ & $-0.05 \pm 0.00_{-0.00}^{+0.00}$ & $-1.11 \pm 0.00_{-0.00}^{+0.000}$ & $-2.56 \pm 0.01_{-0.01}^{+0.01}$ & $-1.07 \pm 0.00_{-0.00}^{+0.00}$ & $-2.52 \pm 0.00_{-0.01}^{+0.01}$ & 0.30 \\
\hline V & 6 & - & $0.33 \pm 0.00_{-0.00}^{+0.00}$ & $-0.82 \pm 0.00_{-0.01}^{+0.00}$ & $-2.19 \pm 0.00_{-0.02}^{+0.01}$ & $-1.15 \pm 0.01_{-0.01}^{+0.00}$ & $-2.52 \pm 0.01_{-0.02}^{+0.01}$ & 0.24 \\
\hline V & 7 & $0.90 \pm 0.03$ & $0.09 \pm 0.00_{-0.00}^{+0.00}$ & $-1.01 \pm 0.00_{-0.01}^{+0.00}$ & $-2.31 \pm 0.00_{-0.02}^{+0.00}$ & $-1.10 \pm 0.00_{-0.01}^{+0.00}$ & $-2.39 \pm 0.00_{-0.01}^{+0.00}$ & 0.24 \\
\hline V & 9 & $0.98 \pm 0.03$ & $0.04 \pm 0.00_{-0.00}^{+0.00}$ & $-1.07 \pm 0.00_{-0.01}^{+0.00}$ & $-2.51 \pm 0.01_{-0.02}^{+0.01}$ & $-1.11 \pm 0.00_{-0.01}^{+0.00}$ & $-2.55 \pm 0.01_{-0.02}^{+0.01}$ & 0.24 \\
\hline$R$ & 3 & - & $0.52 \pm 0.00_{-0.00}^{+0.00}$ & $-0.66 \pm 0.00_{-0.00}^{+0.01}$ & $-2.15 \pm 0.00_{-0.01}^{+0.04}$ & $-1.18 \pm 0.00_{-0.00}^{+0.01}$ & $-2.68 \pm 0.01_{-0.01}^{+0.04}$ & 0.13 \\
\hline$R$ & 4 & $1.00 \pm 0.02$ & $0.53 \pm 0.01_{-0.00}^{+0.00}$ & $-0.66 \pm 0.00_{-0.00}^{+0.01}$ & $-2.15 \pm 0.00_{-0.01}^{+0.04}$ & $-1.20 \pm 0.01_{-0.00}^{+0.01}$ & $-2.68 \pm 0.01_{-0.01}^{+0.04}$ & 0.13 \\
\hline$R$ & 6 & $0.99 \pm 0.04$ & $0.41 \pm 0.00_{-0.00}^{+0.00}$ & $-0.76 \pm 0.00_{-0.00}^{+0.01}$ & $-2.14 \pm 0.00_{-0.01}^{+0.04}$ & $-1.17 \pm 0.00_{-0.00}^{+0.01}$ & $-2.55 \pm 0.00_{-0.01}^{+0.04}$ & 0.13 \\
\hline$R$ & 7 & $0.90 \pm 0.04$ & $0.14 \pm 0.00_{-0.00}^{+0.00}$ & $-0.96 \pm 0.01_{-0.00}^{+0.01}$ & $-2.23 \pm 0.00_{-0.01}^{+0.04}$ & $-1.10 \pm 0.01_{-0.00}^{+0.01}$ & $-2.38 \pm 0.00_{-0.01}^{+0.04}$ & 0.13 \\
\hline$R$ & 9 & $0.95 \pm 0.01$ & $0.10 \pm 0.00_{-0.00}^{+0.00}$ & $-1.00 \pm 0.00_{-0.00}^{+0.01}$ & $-2.43 \pm 0.01_{-0.01}^{+0.05}$ & $-1.10 \pm 0.01_{-0.00}^{+0.02}$ & $-2.53 \pm 0.01_{-0.01}^{+0.05}$ & 0.13 \\
\hline$I$ & 9 & - & $0.01 \pm 0.00_{-0.00}^{+0.00}$ & $-1.01 \pm 0.01_{-0.06}^{+0.01}$ & $-2.29 \pm 0.01_{-0.05}^{+0.07}$ & $-1.02 \pm 0.01_{-0.08}^{+0.01}$ & $-2.30 \pm 0.01_{-0.04}^{+0.05}$ & 0.30 \\
\hline$J$ & 1 & - & $0.34 \pm 0.00_{-0.01}^{+0.02}$ & $-0.64 \pm 0.01_{-0.01}^{+0.01}$ & $-2.17 \pm 0.02_{-0.03}^{+0.06}$ & $-0.99 \pm 0.01_{-0.01}^{+0.02}$ & $-2.51 \pm 0.02_{-0.02}^{+0.04}$ & 0.29 \\
\hline$J \# 5133$ & 8 & - & $-0.01 \pm 0.01_{-0.00}^{+0.00}$ & $-0.84 \pm 0.01_{-0.06}^{+0.04}$ & $-1.78 \pm 0.01_{-0.04}^{+0.06}$ & $-0.84 \pm 0.01_{-0.06}^{+0.04}$ & $-1.78 \pm 0.01_{-0.04}^{+0.06}$ & 0.21 \\
\hline$F 160 W$ & 7 & - & $-0.28 \pm 0.01_{-0.13}^{+0.02}$ & $-1.16 \pm 0.01_{-0.10}^{+0.17}$ & $-2.57 \pm 0.05_{-0.05}^{+0.05}$ & $-0.87 \pm 0.01_{-0.03}^{+0.19}$ & $-2.28 \pm 0.05_{-0.02}^{+0.01}$ & 0.08 \\
\hline H\#5209 & 8 & - & $-0.21 \pm 0.01_{-0.00}^{+0.00}$ & $-0.76 \pm 0.01_{-0.04}^{+0.02}$ & $-1.94 \pm 0.05_{-0.05}^{+0.05}$ & $-0.56 \pm 0.01_{-0.04}^{+0.02}$ & $-1.73 \pm 0.06_{-0.05}^{+0.05}$ & 0.21 \\
\hline$K \mathrm{~s}$ & 2 & - & $-0.39 \pm 0.00_{-0.00}^{+0.02}$ & $-1.00 \pm 0.01_{-0.01}^{+0.01}$ & $-2.46 \pm 0.00_{-0.01}^{+0.03}$ & $-0.61 \pm 0.01_{-0.04}^{+0.01}$ & $-2.06 \pm 0.00_{-0.04}^{+0.03}$ & 0.21 \\
\hline$K^{\prime} \# 5337$ & 8 & - & $-0.41 \pm 0.01_{-0.03}^{+0.03}$ & $-1.01 \pm 0.01_{-0.05}^{+0.01}$ & $-2.28 \pm 0.04_{-0.01}^{+0.08}$ & $-0.60 \pm 0.02_{-0.07}^{+0.04}$ & $-1.87 \pm 0.04_{-0.06}^{+0.02}$ & 0.21 \\
\hline
\end{tabular}

\subsubsection{Relative photometry}

We report in this section the flux ratios between the lensed images deduced from the multi-epoch deconvolution when possible and from single epoch data otherwise (cf. Sect. 3.1).

Multi-epoch deconvolution consists in deconvolving simultaneously a set of frames obtained in a filter at several epochs. We report the results obtained for $B-, V-$ and $R-$ band data ${ }^{5}$ at 3,3 and 5 epochs. For all epochs and all bands, except

\footnotetext{
5 Although $J(K)$ band data exist at two epochs, the filter response curves are significantly different. Flux variations are thus less reliable and not reported.
}

epoch 6 in the $V$ band, all individual frames have been used. For epoch 6 in the $V$ band, we only included the 5 best-seeing frames. For each filter, we rebinned the FORS1 frames to the sampling of the FORS2 frames. Then, we applied frame to frame zero-point correction calculated based on photometric measurements of common stars in each field. Then, we ran MCS on the registered data and derived the magnitude differences and flux time variations. Due to a small apparent systematic bias ( $~ 0.01 \mathrm{mag}$ ) of the MCS photometry, we corrected our measurements based on the comparison of the aperture photometry and MCS photometry of 4 field stars. Final results are presented in Table 4 (11 first rows). 
The reduction procedure of single epoch data has already been described in Sect. 3.3.1. Only CFHT-IR data have not yet been discussed. For those, the complete set of individual frames have been simultaneously deconvolved with a final FWHM of $0.21^{\prime \prime}$.

The full results are reported in Table 4. The photometric (random) errors were calculated based on the dispersion of the measurements obtained for the different frames. Following this method, the Poisson noise and the error coming from the imperfection of the kernel used for deconvolution are included in the error bars. This is confirmed by the fact that estimated errors are larger or equal to photon noise. The systematic error was estimated from the results obtained for $\lambda_{\text {low }}$ and $\lambda_{\text {high }}$ (cf. Sect. 3.1). We observe, between these two limits, typical variations of the flux ratio by 5 up to $15 \%$ from the $V$ to the $K$ bands while it is $\leq 2 \%$ in the $B$ band for which the contrast between the ring and the lensed QSO images is maximum. At a given wavelength, these systematic errors are reduced when the S/N in the Einstein ring increases.

Finally, note that whenever possible, we have compared the flux ratios deduced during the multi-epoch process to single epoch deconvolution results. Both measurements are basically identical $^{6}$ but multi-epoch results are less sensitive to systematic errors because of the increased $\mathrm{S} / \mathrm{N}$ in the ring that improves the separation between point-like and extended flux.

\subsubsection{Caveat}

Because of the presence of the Einstein ring superimposed over the quasar images, one may ask whether the measured pointlike fluxes are associated with the quasar only (i.e. compact emission) or whether they are significantly mixed with emission originating from a more spatially extended (partly unresolved) region of the host galaxy. In the following we examine how the core of the host galaxy may modify the observed fluxes of images A, B and C.

The observed flux ratios between images $\mathrm{A}, \mathrm{B}, \mathrm{C}$ are contaminated in two ways by the ring.

The first one is a purely instrumental effect. The resolved ring plays the role of a spatially variable background, whose intensity is increasing below the unresolved PSF because this region corresponds to the innermost parts of the host. However the deconvolution process tends to produce a "flat" background below the PSF whose intensity closely matches the value in its close vicinity. Since the surface brightness of the resolved background ring is the same around each point-like image, the flux of the faint images (i.e. weakly amplified) is more contaminated by the ring than the flux of the bright ones. Consequently, the flux ratios between brighter and weaker lensed images are underestimated with respect to the theoretical values. This ring contamination increases with the ring brightness (i.e. from the $B$ band to the $K$ band) and we call this the background effect.

The second effect is related to the specific geometry of $\mathrm{J} 1131$ in the source plane. Indeed, according to the lens models, the QSO core is located at only $0.016^{\prime \prime}(115 \mathrm{pc}$ in the source

\footnotetext{
${ }^{6}$ Optical single epoch magnitude difference can be inferred for epoch 9 in Table 3.
}

plane; Paper I) from a macro caustic. Hence, the dusty torus and the most central part of the host galaxy (that contributes significantly to the NIR flux) cover a region in the source plane with a strong magnification gradient. Therefore, the amplifications of the lensed images will depend on the considered source radius even at very small, unresolved scales. Moreover, since the local magnification around image A is larger than around images B \& $\mathrm{C}$, the unresolved source radius will be smaller for image A and the amplification ratios will also be affected. We dub this effect the differential amplification effect.

Although the latter effect depends on the radial distribution of the source light, which is itself a function of wavelength, we made simulations to quantify its trend. We first chose a circularly symmetric exponential light profile with a half-flux radius $r_{\mathrm{e}}=1.9 \mathrm{kpc}\left(=0.25^{\prime \prime}\right)$ to represent a typical quasar host galaxy (Sánchez et al. 2004). We lensed that extended source, sampled on a grid of $0.00025^{\prime \prime} /$ pixel, with a Singular Isothermal Ellipsoid + shear model fitting our data (SIE $+\gamma$; Sect. 4.2) using the gravlens software v1.06 (Keeton 2001). The image plane, sampled on a 5 mas grid, was convolved with a Moffat profile $\left(F W H M=0.7^{\prime \prime}\right)$ and rebinned to a scale of $0.16^{\prime \prime} /$ pixel to mimic the observations. This synthetic frame was then deconvolved using the MCS technique to a final seeing of $F W H M=0.32^{\prime \prime}$. We repeated this operation for several source radii in the range $0.005^{\prime \prime}<r_{\mathrm{src}}<0.1^{\prime \prime}$ and we computed the flux ratios $I_{\mathrm{A}} / I_{\mathrm{B}}$ and $I_{\mathrm{A}} / I_{\mathrm{C}}$. The main result is that the source remains unresolved in the A, B and C images for $r_{\text {src }}<0.015^{\prime \prime}$. The associated flux ratios are then nearly identical to the point-like source ones $(\Delta m \sim 0.05 \mathrm{mag})$. For larger source radii, the images become resolved and these flux ratios decrease and converge towards 1.

Finally, we simulated a more realistic source model with two components: an extended exponential profile (truncated to $\left.r_{\text {host }}=0.1^{\prime \prime}\right)$ lensed into a resolved background ring and a superimposed disk-like source $\left(r_{\mathrm{QSO}}=0.01^{\prime \prime}\right)$ lensed into unresolved images. This enabled us to look for the joined influence of the background effect and the differential amplification effect on the observed magnitude differences between the unresolved images. We quantify the effect as a function of the host contamination (defined as the fraction of the total point-like flux coming from the host galaxy). The results are displayed in Fig. 1.

Because the QSO Spectral Energy Distribution (SED) is expected to be dimmer than the host SED in the NIR range, we may expect a stronger ring contamination in that range. An accurate estimate would require us to know the true SEDs, but we can already infer an approximate contamination from the existing data. Indeed, in the $B$ band, the ring image is faint and we estimate the host contribution to be at maximum $5 \%$ of the total point-like flux. Using a mean color index $B-K=2.5$ for the QSO and $B-K=5$ for the host galaxy, we calculate that in the $K$ band up to $35 \%$ of the total point-like flux is coming from the host. Reporting these values in Fig. 1, and comparing them with the case of $0 \%$ contamination, we derive that the observed flux ratios should not deviate by more than 0.05 mag from the predictions for a point-like source in the $B$ band. In the $K$ band, $\Delta m_{\mathrm{AB}}$ and $\Delta m_{\mathrm{AC}}$ are reduced by several tenths of magnitude. On the contrary, $\Delta m_{\mathrm{BC}}$ does not vary significantly. 


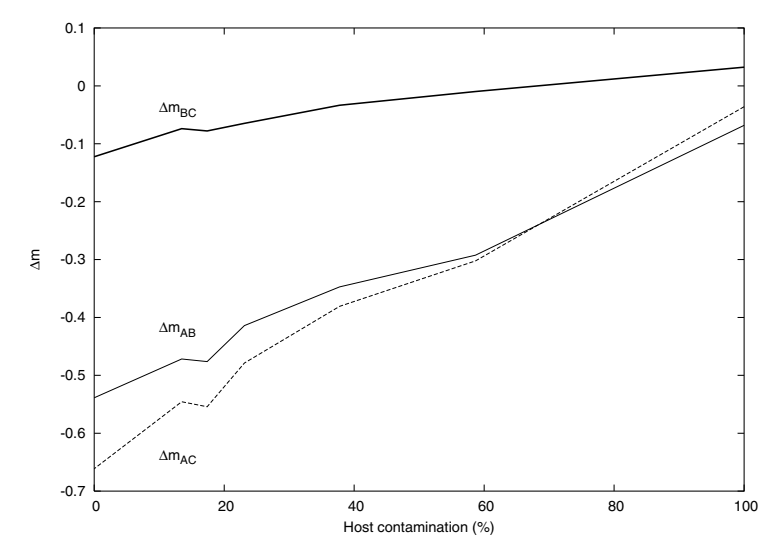

Fig. 1. Simulated point-like magnitude difference $\Delta m_{\mathrm{AB}}$ (thin solid line), $\Delta m_{\mathrm{AC}}$ (dotted line) and $\Delta m_{\mathrm{BC}}$ (bold solid line) as a function of the average fraction of the flux of the host galaxy included in the total point-like flux after deconvolution (host contamination).

\section{Results}

\subsection{Ring morphology}

The multi-wavelength images of J1131 confirm that the contrast between the QSO and its host decreases with increasing wavelength, as expected for the host galaxy of a quasar located at $z \sim 0.66$. Indeed, the SED of QSOs is minimum at $\sim 1-2 \mu \mathrm{m}$ (rest-frame) while in this range the dominant emission is coming from the host (e.g. Dunlop et al. 1993) and marginally from the dust torus (hot dust emission increases up to $\sim 2 \mu \mathrm{m}$; e.g. Elvis et al. 1994; Nenkova et al. 2002).

While some structures in the ring are barely detected on the ground based $K$ s deconvolved frames (Fig. 2, right), the HST NIC2 deconvolved image (Fig. 2, left) spectacularly unveils many details in the ring. Additionally, a putative companion object $(X)$ near the lensing galaxy is more clearly detected. Its nature is investigated in Paper II based on ACS and NICMOS data and it is shown that the expected influence of $X$ on the lens modeling can only be very weak.

\subsection{Lens modeling}

In this section we construct a fiducial lens model aimed at discussing the observed flux ratios and estimating the time delays for J1131. This adopted lens model is a Singular Isothermal Ellipsoid (SIE) + external shear $(\gamma)$, which is generically used in the study of the flux ratio anomalies (Dobler \& Keeton 2005, and references therein). It has also the advantage to be fully constrained by the astrometry of $\mathrm{J} 1131$ (i.e. the positions of A, B, C, D and G). Additionally, it takes into account the main characteristics of the deflector, namely the lens galaxy ellipticity and the external shear associated with the lens environment. We used the gravlens modeling package v 1.06 (Keeton 2001) constrained by the HST relative astrometry of the pointlike images and of the lensing galaxy (Sect. 3.2). Since systematic errors affecting the NICMOS data (error on the plate scale, on the distorsion, on the orientation and due to PSF undersampling) do not influence similarly all lensed images, we have included them in the position error bars. The model yields the following results: $\theta_{\mathrm{E}}=1.843^{\prime \prime}, e=0.162, \theta_{e}=-57.92^{\circ}$, $\gamma=0.114$ and $\theta_{\gamma}=-83.01^{\circ}$; where $\theta_{\mathrm{E}}$ is the Einstein angular radius, $e$ is the ellipticity and $\theta_{e}$ is its PA, $\gamma$ is the tidal shear and $\theta_{\gamma}$ (counted $\mathrm{E}$ of $\mathrm{N}$ ) points towards the mass producing the shear. We should note that the shear does not point towards any obvious massive perturber in the field. On the other hand $e$ and $\theta_{e}$ agree well with the light profile ellipticity and PA of the lens galaxy (see Paper II). The reduced $\chi^{2}$ (for 1 d.o.f.) associated with this model amounts to 136 which is statistically unacceptable. The disagreement between the model predictions and the observations is mainly caused by the lensing galaxy position $\left(\chi_{\text {gal }}^{2}=90\right)$ expected $0.03^{\prime \prime}$ South from its observed position (Table 5).

Assuming that we can ignore $\mathrm{X}$ in the modeling, we opted for the SIE $+\gamma$ model as our fiducial lens model ${ }^{7}$. This model leads to predicted time delays between $\mathrm{C}$ (leading) and the other multiple images: $\tau_{\mathrm{CB}}=0.3 \mathrm{~d}, \tau_{\mathrm{CA}}=$ $1.3 \mathrm{~d}$ and $\tau_{\mathrm{CD}}=126.9 \mathrm{~d}$. The predicted flux ratios are reported in Table 5. We have also fitted an ensemble of non parametric asymmetric models (searched by a Monte-Carlo method) with the PixeLens publicly available software (Saha $\&$ Williams 2004). This enabled us to derive a distribution of $\tau_{\mathrm{CD}}$ (each $\tau_{\mathrm{CD}}$ being associated with a different model) in the range $80-400$ days while $\tau_{\mathrm{CA}}$ may not exceed 5 days.

\subsection{Flux variability}

\subsubsection{Temporal flux ratio variations}

Flux variations of the lensed images of J1131 have been obtained in Sect. 3.3.2. Figure 3 represents the corresponding lightcurves in the $R$ band $^{8}$. The comparison of these ones with the lighcurves observed in the $V$ and $B$ bands (i.e. epochs 6-7-9 in $V$ and epochs 7 and 9 in $B$ ) indicates an agreement between the lightcurves within $0.05 \mathrm{mag}$ and often around $0.02 \mathrm{mag}$. Lightcurves are also nearly insensitive to the choice of the regularization parameter (except in the $R$ band where the maximum shift of the D lightcurve can reach $0.05 \mathrm{mag}$ ).

Figure 3 reveals a dimming of $B$ and $C$ by about $0.3 \mathrm{mag}$ in an eleven month period. A dimming of $\mathrm{D}$ is observed with a possible delay of about 5 months. During this period, the flux of A changed by only $\sim 0.1 \mathrm{mag}$.

Because the time-delay between A-B-C is of the order of a few days, intrinsic flux variations of the source would produce nearly simultaneous flux variations between these images. Therefore, the different behaviour of image A with respect to $B$ and $\mathrm{C}$ suggests that a microlensing event occurs in image $\mathrm{A}$ (i.e. microlensing partially compensates the intrinsic flux variations; scenario S1). Alternatively, B and C might be dimmed by two independent microlensing events producing nearly simultaneously the same lightcurve for both images (scenario S2).

\footnotetext{
${ }^{7}$ We also emphasize that SIE $+\gamma$ left us with only 1 d.o.f. More complex models thus need additional constraints (see Paper II).

8 One might suspect that the difference of response between the Special $R$ filter (epochs 3, 4, 6, 7) and the Bessel $R$ filter (epoch 9) biases the observed lightcurve. But the color correction only amounts to 0.02 mag for the spectrum of J1131 and should not significantly modify our results.
} 

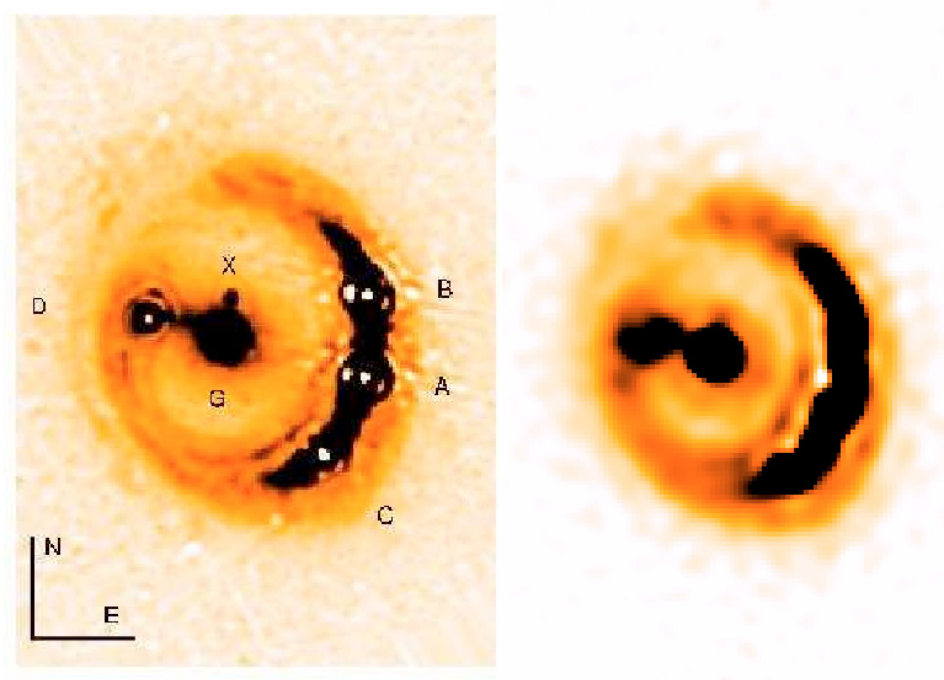

Fig. 2. Left: deconvolved HST NICMOS $F 160 W$ image mainly showing the ring. $F W H M=0.08^{\prime \prime}$. Right: deconvolved image in the $K$ s filter obtained with ISAAC. $F W H M=0.15^{\prime \prime}$.

Table 5. Summary of the observed relative astrometry and photometry related to J1131 and comparison with model predictions. The observed relative positions with respect to image A $(o b s)$, obtained from NICMOS images, are those given in Table 2. The systematic errors on the relative positions have been quadratically added to the random errors affecting the measurements. Since the position of A is not fixed to the observed one but known with the uncertainty reported in Table 2, the modeled position of A is not $(0,0)$. Flux ratios $I_{i} / I_{j}$ in the $K$ band are reported when microlensing (scenario $\mathrm{S} 1$ or $\mathrm{S} 2$ ) affects image $i$ or $j$ and in the $B$ band otherwise. Since microlensing is minimum in the $K$ band, the flux ratio reported is a lower/upper limit on the "true flux ratio". Flux ratios including image D are placed into brackets because they are not corrected for the unknown time delay. Additionally, a microlensing event may also occur for that image.

\begin{tabular}{|c|c|c|c|c|c|c|c|c|}
\hline ID (Parity) & \multicolumn{2}{|c|}{$\Delta \alpha \cos \delta\left(^{\prime \prime}\right)$} & \multicolumn{2}{|c|}{$\Delta \delta\left(^{\prime \prime}\right)$} & \multicolumn{2}{|l|}{$I_{\mathrm{B}} / I_{j}$} & \multicolumn{2}{|l|}{$I_{\mathrm{A}} / I_{j}$} \\
\hline & obs & mod-obs & obs & mod-obs & S1 & $\bmod$ & S2 & $\bmod$ \\
\hline$A(-)$ & $0.000 \pm 0.003$ & -0.002 & $0.000 \pm 0.003$ & -0.002 & $\leq 0.68$ & 0.61 & 1.00 & 1.00 \\
\hline $\mathrm{B}(+)$ & $0.032 \pm 0.003$ & -0.002 & $1.184 \pm 0.003$ & 0.003 & 1.00 & 1.00 & $\geq 1.46$ & 1.65 \\
\hline $\mathrm{C}(+)$ & $-0.590 \pm 0.003$ & 0.002 & $-1.117 \pm 0.003$ & 0.003 & $2.67 \pm 0.01$ & 1.11 & $\geq 2.53$ & 1.83 \\
\hline $\mathrm{D}(-)$ & $-3.115 \pm 0.003$ & 0.006 & $0.875 \pm 0.004$ & 0.019 & {$[10.02 \pm 0.01]$} & 11.95 & {$[10.58 \pm 0.06]$} & 19.72 \\
\hline $\mathrm{G}$ & $-2.027 \pm 0.003$ & -0.010 & $0.607 \pm 0.004$ & -0.029 & - & - & - & - \\
\hline$X$ & $-1.936 \pm 0.011$ & - & $1.100 \pm 0.011$ & - & - & - & - & - \\
\hline
\end{tabular}

For both $\mathrm{S} 1$ and $\mathrm{S} 2$, the observed microlensing time-scale is compatible with the crossing time of a microcaustic by the source (e.g. Treyer \& Wambsganss 2004). Finally, we note that the dimming of $\mathrm{D}$ five months (i.e. $\sim \tau_{C D}$ ) after $\mathrm{B}$ and $\mathrm{C}$ supports $\mathrm{S} 1$, but microlensing in $\mathrm{D}$ cannot either be excluded.

\subsubsection{Chromatic variations}

Three phenomena can a priori introduce chromatic effects in the observed flux ratios: $(i)$ differential extinction produced by dust in the lensing galaxy and which is expected to mainly affect the blue bands; (ii) microlensing, which is stronger for smaller source sizes (e.g.Wambsganss \& Paczyński 1991; Wyithe et al. 2000) and therefore for the shorter wavelengths emitted in the most central parts of the accretion disk (in the NIR, the microlensing signal would also be swamped by the constant flux contribution from the host); (iii) systematic errors due to the differential amplification and to the background effect which decrease by several tenths the modulus of the magnitude difference between two images from B to K (Sect. 3.3.3 and Fig. 1).

Figure 4 shows the magnitude differences between the point-like images as seen through different filters from the $B$ to the $K$ band, at epochs 8 (NIR range) and 9 (optical range). Chromatic variations are low (i.e. $\leq 0.15 \mathrm{mag}$ ) in the optical while variations up to $0.5 \mathrm{mag}$ are observed within the NIR range. This first statement does not argue in favor of strong differential extinction between lensed images since this one should be stronger in the optical range (where effect $i i i$ is negligible) than in the NIR. Differential reddening can thus be safely neglected in the following.

We now interpret the chromaticity of $\Delta m_{\mathrm{AB}}, \Delta m_{\mathrm{BC}}$ and $\Delta m_{\mathrm{AC}}$ (magnitude differences involving $\mathrm{D}$ are not discussed because of the large unknown time delay for this image) between optical and NIR wavelengths. Note that in the following, we discuss the chromatic variations for optical and NIR range 


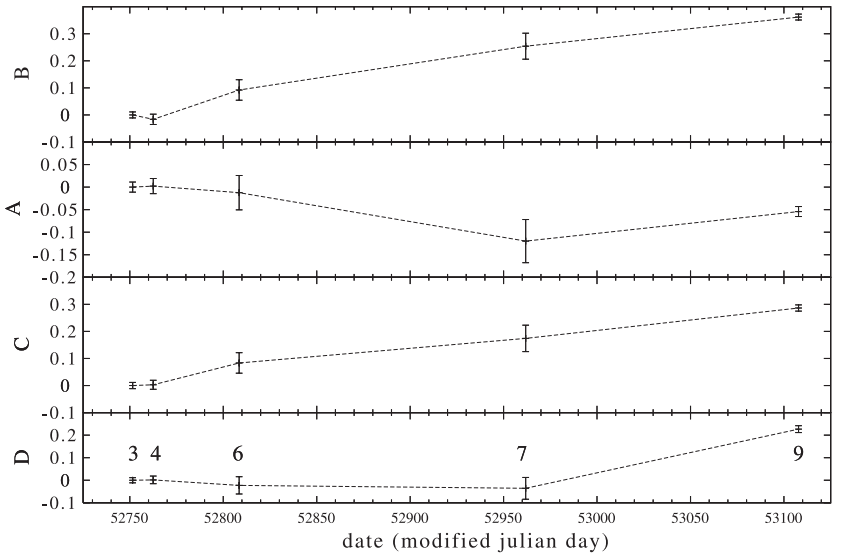

Fig. 3. Lightcurves of images B, A, C and D in the $R$ band for epochs 3 , $4,6,7,9$. The $x$ axis refers to the observing date in modified julian day (i.e. julian day-2 400000 ). The $y$ axis refers to the magnitude difference with respect to epoch 3 (i.e. $m_{i}-m_{3}$ ). The dotted line joining the points is plotted for the legibility of the graph.

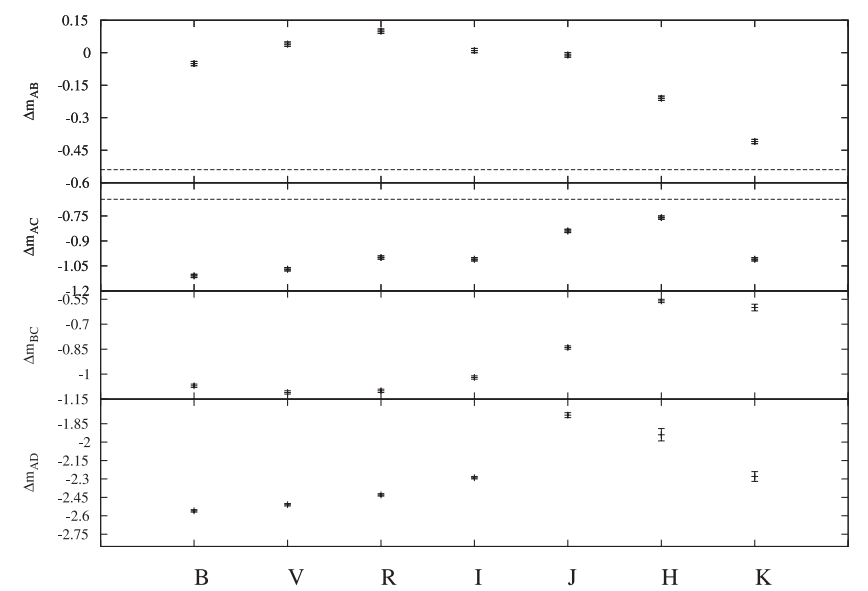

Fig. 4. Magnitude differences between A (or B) and the other pointlike images in $B, V, R, I$ as observed at epoch 9 and in $J, H, K$ at epoch 8 (cf. Table 4). The dotted line, when visible, represents the flux ratio predicted by the SIE $+\gamma$ model, namely $\Delta m_{\mathrm{AB}}=-0.54, \Delta m_{\mathrm{AC}}=$ $-0.65, \Delta m_{\mathrm{BC}}=-0.11$ and $\Delta m_{\mathrm{AD}}=-3.23 .1 \sigma$ error bars are displayed.

separately when microlensing might have produced flux ratio variations between epochs 8 - NIR - and 9-optical -:

- $\Delta m_{\mathrm{AB}}$ : Fig. 4 shows that $\Delta m_{\mathrm{AB}} \sim 0$ mag in the optical and decreases down to $-0.45 \mathrm{mag}$ in the $K$ band. Both microlensing scenarii described in Sect. 4.3.1 can reproduce the fact that $\left|\Delta m_{\mathrm{AB}}\right|$ is smaller in the $J$ band than in the $K$ band: either $\mathrm{A}$ is affected ( $\mathrm{S} 1)$ and is then de-amplified or B is affected (S2) and is then amplified. Note that we do not expect chromatic variation caused by phenomenon iii since $\Delta m_{\mathrm{AB}} \sim 0 \mathrm{mag}$ in the $J$ band. Whatever the adopted scenario, the value of $I_{\mathrm{B}} / I_{\mathrm{A}}$ observed in the $K$ band is the least affected by microlensing and thus constitutes an upper limit on the true flux ratio $I_{\mathrm{B}} / I_{\mathrm{A}}$ (Table 5).

- $\Delta m_{\mathrm{BC}}:\left|\Delta m_{\mathrm{BC}}\right| \sim 1 \mathrm{mag}$ in the optical range and decreases to $\left|\Delta m_{\mathrm{BC}}\right| \sim 0.55 \mathrm{mag}$ in the $H$ and $K$ bands. Under $\mathrm{S} 1$, this fading of $I_{\mathrm{B}} / I_{\mathrm{C}}$ from the $B$ to the $K$ band can be explained by effect $i i i$ (since $\left|\Delta m_{\mathrm{BC}}\right|$ differs significantly from
0 and $\mathrm{B}$ and $\mathrm{C}$ are not microlensed). Note that since $\tau_{\mathrm{CB}}$ is very short and since the ring contamination is negligible in the $B$ band, $\Delta m_{\mathrm{BC}}$ in the $B$ band should correctly represent the macro-lens flux ratio under $\mathrm{S} 1$. However, this value $\left(\Delta m_{\mathrm{BC}}=-1.05 \mathrm{mag}\right)$ is abnormally low compared to the value predicted by the model $\left(\Delta m_{\mathrm{BC}}^{\text {model }}=-0.11 \mathrm{mag}\right)$. Under scenario $\mathrm{S} 2$, several combinations of microlensing for $\mathrm{B}$ and $\mathrm{C}$ and effect $i i i$ are possible.

- $\Delta m_{\mathrm{AC}}:\left|\Delta m_{\mathrm{AC}}\right| \sim 1.05 \mathrm{mag}$ in the optical range. It amounts to $0.84 \mathrm{mag}$ in the $J$ band, $0.76 \mathrm{mag}$ in the $H$ band and rises up to $1.01 \mathrm{mag}$ in the $K$ band. First under $\mathrm{S} 1$, we can explain the observed decrease of $\left|\Delta m_{\mathrm{AC}}\right|$ between the $J$ and $H$ bands by the dominant ring contamination, while the subsequent increase of $\left|\Delta m_{\mathrm{AC}}\right|$ in the $K$ band would be due to the drop of the microlensing de-amplification of image $\mathrm{A}$. Under $\mathrm{S} 2$, the same interpretation remains valid except that the increase of $\left|\Delta m_{\mathrm{AC}}\right|$ in the $K$ band implies that image $\mathrm{C}$ is more amplified by microlensing at bluer wavelengths. In the latter case, its value in the $K$ band should be a lower limit of the macro-lens flux ratio. This reveals an abnormally large value of $\left|\Delta m_{\mathrm{AC}}\right|$ (compared to the fiducial model prediction) under scenario S2.

In summary, our data first suggest the absence of strong differential extinction between the lensed images of J1131. Second, the observed chromatic variations of the flux ratios can qualitatively be explained by the combination of effects $i i$ and iii. More interestingly, this analysis also reveals that either $\mathrm{A}$ is de-amplified by a microlensing event (S1) or B and C are both amplified by two independent microlensing events (S2). For both microlensing scenarii, we have explained in which filter the flux ratio measurement should be the closest to the values predicted by the "macro model". As one can see in Table 5, these "best" estimates of the flux ratios do not reconcile with the macro model predictions. We investigate how anomalous are the flux ratios in the next section.

\subsubsection{Anomalous flux ratios}

Anomalous flux ratios in lensed systems have caught little attention until the work of Mao \& Schneider (1998) who first realised that substructures could significantly modify the flux ratios in $\mathrm{B} 1422+231$. Various authors tried to compare the amount of Dark Matter Halo substructures predicted by $\Lambda C D M$ hydrodynamical simulations to the amount of substructures necessary in lensing models to reproduce observed flux ratios (e.g. Metcalf \& Madau 2001; Chiba 2002; Dalal \& Kochanek 2002; Metcalf \& Zhao 2002; Mao et al. 2004; Bradač et al. 2002, 2004; Kochanek \& Dalal 2004; Metcalf 2005a). However, it is still unclear whether the amount of substructures necessary in lensing models agrees or not with the hydrodynamical simulation predictions (see e.g. KGP05 or Rozo et al. 2005 for a recent extensive summary of the situation).

Indeed, from a lensing perspective, flux ratios are not only sensitive to substructures but also to $m=3$ and $m=4$ multipoles of the lens model (Evans \& Witt 2003; Möller et al. 2003; Quadri et al. 2003; Kawano et al. 2004), to the 
lens environment (Oguri 2005), to the source size (Dobler \& Keeton 2005; Inoue \& Chiba 2005; Chiba et al. 2005) and to isolated dark matter clumps on the same line of sight (Chen et al. 2003; Metcalf 2005a,b; Wambsganss et al. 2005). From a hydrodynamical simulation perspective, the expected amount of dark matter within the Einstein radius of a lens is not well known. Indeed, both the abundance of halo substructures (e.g. Zentner \& Bullock 2002; Sigurdson \& Kamionkowski 2004) and their spatial distribution (e.g. Chen et al. 2003; Zentner \& Bullock 2003; De Lucia et al. 2004; Oguri \& Lee 2004) are uncertain. In particular the amount of dark matter clumps that survives in the central part of a halo (and so within a lens Einstein radius) is still debated (e.g. Mao et al. 2004; Amara et al. 2004; Trott \& Melatos 2005; Macciò et al. 2005).

In any case, the first step of any analysis is to obtain secure flux ratios and to identify anomalies independently of the choice of a macro-lens model. For this purpose, the most reliable method is based on the validity/violation of the so called "magnification sum rules", originally introduced by Schneider \& Weiss (1992). The first rule predicts that a pair of images created when the source crosses a fold caustic will have the same brightness. The second one is valid when an unresolved source lies near a cusp caustic and predicts that the quantity $R_{\text {cusp }}$ (Mao \& Schneider 1998):

$R_{\text {cusp }}=\frac{\left|\mu_{\mathrm{A}}+\mu_{\mathrm{B}}+\mu_{\mathrm{C}}\right|}{\left|\mu_{\mathrm{A}}\right|+\left|\mu_{\mathrm{B}}\right|+\left|\mu_{\mathrm{C}}\right|}=\frac{\left|1-I_{\mathrm{B}} / I_{\mathrm{A}}-I_{\mathrm{C}} / I_{\mathrm{A}}\right|}{1+I_{\mathrm{B}} / I_{\mathrm{A}}+I_{\mathrm{C}} / I_{\mathrm{A}}}$,

converges towards 0 as the source gets closer to a cusp singularity and all the three images are merging.

The latter relation appears to be more robust than the relation binding "fold images" (KGP03, KGP05). Cusp configuration systems are thus particularly important for the study of flux ratio anomalies (KGP03, Bradač 2004). Among those, only two systems possess radio flux ratios $(\mathrm{B} 1422+231$ and B2045+265 ${ }^{9}$ ). The two others (i.e. RXJ 0911+0551 and J1131) could only be observed in the optical range and may thus be contaminated by dust extinction and microlensing. Hereafter, we further discuss the case of $\mathrm{J} 1131$ for which $R_{\text {cusp }} \sim 0.35$ at the epoch of the discovery (e.g. KGP03).

Although microlensing precludes the determination of the true flux ratios, we have shown in Sect. 4.3.2 that reliable upper/lower limits on these ones could be derived (Table 5). Using the flux ratios of Table 5, we find that $R_{\text {cusp }} \geq 0.036$ whatever the microlensing scenario. Although $R_{\text {cusp }}$ might naturally deviate from 0 (KGP03), the observed value is fully consistent with no violation of the cusp relation. This does not automatically mean that effects of substructures are rejected. First, we only have a lower limit on $R_{\text {cusp }}$ and second, "small" $R_{\text {cusp }}$ values can be observed while flux ratios cannot be reproduced by realistic lens models (cf. B1422+231; KGP03; Bradač et al. 2002).

As already noticed in Sect. 4.3.2, $I_{\mathrm{B}} / I_{\mathrm{C}}\left(I_{\mathrm{A}} / I_{\mathrm{C}}\right)$ does not agree with our fiducial model predictions under S1 (S2). Remembering that substructures can only magnify a minimum of the arrival time surface and preferentially demagnify saddle points (e.g. Schechter \& Wambsganss 2002), we now look if substructures are able to explain that flux ratio. Under scenario $\mathrm{S} 1, I_{\mathrm{B}} / I_{\mathrm{C}}=2.67$ while the predicted value is 1.11

\footnotetext{
9 We do not consider the hybrid fold/cusp case of B0742+472.
}

(Table 5). Both $\mathrm{B}$ and $\mathrm{C}$ are minima of the arrival time surface. Consequently, only a magnification of B by 0.95 mag is allowed by the substructure scenario (since $\mathrm{C}$ being a minimum cannot be de-amplified by milli-lensing) to explain $I_{\mathrm{B}} / I_{\mathrm{C}}$. However, the value of $I_{\mathrm{A}} / I_{\mathrm{C}}\left(I_{\mathrm{A}} / I_{\mathrm{C}} \geq 3.92\right)$ derived under $\mathrm{S} 1$ would then imply that A should be magnified by a factor $\geq 2.15$ by substructures to match the predicted value of $I_{\mathrm{A}} / I_{\mathrm{C}}=$ 1.83 and this is very unlikely because i- $t w o$ substructures are required, ii- the saddle-point image A must be amplified.

Under $\mathrm{S} 2, I_{\mathrm{A}} / I_{\mathrm{C}} \geq 2.53$ while the macro model predicts 1.83. Since image $C$ is located at a minimum of the arrival time surface and, thus, cannot be demagnified by substructures, the above flux ratio can only be explained if image $\mathrm{A}$ is amplified by substructures by more than $0.35 \mathrm{mag}$ (i.e. $2.5 \log (2.53 / 1.83))$. This would also reproduce the observed value of $I_{\mathrm{A}} / I_{\mathrm{B}}$. Nevertheless, since image A is a saddle-point, it has a low probability to be magnified by a substructure.

Consequently, under S1, a single substructure in front of a lensed image cannot explain the observed value of $I_{B} / I_{C}$. Under S2, a milli-lensing magnification of A might explain the observed flux ratios. However, magnification of a saddle point by a substructure is statistically quite unlikely.

\section{Summary and conclusions}

We have presented direct optical and NIR imaging of the quadruply imaged quasar RXS J113155.4-123155 obtained with ground-based telescopes and with HST at various epochs. The HST NICMOS images unveil many details in the Einstein ring of this system as well as a putative companion object (X) lying at $\sim 1^{\prime \prime}$ from the lensing galaxy. Accurate positions have been derived for the 4 lensed images, for $\mathrm{X}$ and for the lensing galaxy based on the HST NIC2 data and on the ground-based VLT observations. Both sets of measurements do agree within 6 mas. A SIE $+\gamma$ model has been fitted to the observed image configuration. Nevertheless, the positions of image D and of the lens galaxy do not match satisfactorily the observed ones. Additionally, we have used the SIE $+\gamma$ model to derive the expected point-like flux ratios when taking into account the extended nature of the source.

The images have been deconvolved using the MCS method known to preserve photometry. Hence, we derived for the first time relative and absolute photometry of J1131 from the optical to the NIR range. Since the photometric measurements of the point-like images in J1131 are complicated by the superimposed Einstein ring, we have thoroughly discussed the potential sources of photometric errors. We have shown that the MCS method allows to reach a photometric accuracy close to the photon noise limit in the $B$ and $V$ filters if a good quality PSF can be constructed. On the other hand, small systematic errors affect the flux ratios in the $R$ band due to the Einstein ring.

The small chromatic variations of the flux ratios between the $B$ and $R$ bands suggest a low level of differential extinction between the lensed images of J1131. Based on sparse photometric measurements obtained between November 2002 and April 2004, we have shown the likely presence of microlensing for image A together with intrinsic quasar flux variability up to 
0.3 mag. Microlensing of A is also supported by our Chandra observations of J1131 in the X-rays (see Paper III, Claeskens et al., in preparation; Blackburne et al. 2005). Additionally, based on the chromatic variations of the flux ratios, we have shown that A should likely be de-amplified (scenario S1), in agreement with the fact that it is a saddle-point of the arrivaltime surface ${ }^{10}$ (Schechter \& Wambsganss 2002; Keeton 2003). Alternatively, our data cannot rule out that two independent microlensing amplifications (scenario S2) with roughly the same amplitude occur for both images B and C.

We have used the $R_{\text {cusp }}$ relation (Mao \& Schneider 1998; Eq. (1)) to investigate the evidence for substructures in the lensing galaxy. Once lower/upper bounds on the flux ratios are imposed from our knowledge of microlensing occuring in this system, we find a lower limit on $R_{\text {cusp }}$ close to zero. This shows that the small scale structure(s) identified in KGP03 from the $R_{\text {cusp }}$ relation, was (were) indeed due to (a) microlens(es).

Nevertheless, the observed flux ratios do not agree with the model predictions. We argue that milli-lensing effects cannot provide the best explanation under S1 while under S2, observations suggest that a massive substructure should magnify the saddle point image A. This is however a low probability scenario (Schechter \& Wambsganss 2002). An alternative to the milli-lensing explanation is to introduce in the macro-lens potential higher order multipole terms (e.g. Evans \& Witt 2003). This is described in Paper II, where we successfully added an octupole $(m=4)$ to the fiducial model and reproduced both the observed flux ratio $I_{\mathrm{B}} / I_{\mathrm{C}}$ (under $\mathrm{S} 1$ ) and the observed relative lens position. Therefore, we found that the conjunction of microlensing and of higher order terms in the lens potential could explain the flux ratios observed in J1131. The preference of a more complex macro model over the presence of substructure is opposite to what Kochanek \& Dalal (2004) have found for other lenses. Of course the solution of adding an octupole might not be unique and other models such as those including several sources of shear (e.g. Morgan et al. 2005), or with two mass components (e.g. Dye \& Warren 2005) should be explored.

Acknowledgements. Dominique Sluse acknowledges support from an ESO studentship in Santiago and PRODEX (Gravitational lens studies with HST). JFC acknowledges support from PRODEX (XMM and HST). Part of the research was also performed in the framework of the IUAP P5/36 project, supported by the OSTC Belgian Federal services. We gratefully thank M. Bremer, M. Pierre and J. Willis who allowed us to perform extra observations of J1131. We also want to thank the referee, Steve Warren, whose comments improved the paper. D.S. wants to thank Thierry Forveille and Audrey Delsanti for their advice in the reduction of the NIR data, Olivier Hainaut for helpful discussions on data reductions (among other things), Frédéric Courbin for providing us the MCS code. Our ISAAC service astronomer, A. Jaunsen, as well

\footnotetext{
${ }^{10}$ During the referee process, Rozo et al. (2005) demonstrated that small scale structures, if they can be considered as linear perturbations, might not preferentially demagnify saddle point images. However, large perturbations from point-mass microlenses are likely caused by non linear perturbers and the results of Rozo et al. (2005) may not be valid in such a case.
}

as the FORS service astronomers are warmly acknowledged for their excellent work.

\section{References}

Amara, A., Metcalf, R. B., Cox, T. J., \& Ostriker, J. P. 2004 [arXiv: astro-ph/0411587]

Bertin, E., \& Arnouts, S. 1996, A\&AS, 117, 393

Blackburne, J. A., Pooley, D., \& Rappaport, S. 2005, [arXiv: astro-ph/0509027]

Bradač, M., Schneider, P., Steinmetz, M., et al. 2002, A\&A, 388, 373

Bradač, Schneider, P., Lombardi, M., Steinmetz, M., Koopmans, L.V.E., \& Navarro, J. F. 2004, A\&A, 423, 797

Burud, I., Stabell, R., Magain, P., et al. 1998, A\&A, 339, 701

Burud, I., Hjorth, J., Jaunsen, A. O., et al. 2000, ApJ, 544, 117

Chen, J., Kravtsov, A. V., \& Keeton, C. R. 2003, ApJ, 592, 24

Chiba, M. 2002, ApJ, 565, 17

Chiba, M., Minezaki, T., Kashikawa, N., Kataza, H., \& Inoue, K. T. 2005, ApJ, 627, 53

Claeskens, J. F., Sluse, D., Riaud, P., \& Surdej, J. 2006, A\&A, accepted (Paper II)

Claeskens, J. F., et al., in preparation (Paper III)

Courbin, F., Magain, P., Keeton, C. R., et al. 1997, A\&A, 324, 1

Courbin, F., Lidman, C., Frye, B. L., et al. 1998, ApJ, 499, L119

Dalal, N., \& Kochanek, C. S. 2002, ApJ, 572, 25

De Lucia, G., Kauffmann, G., Springel, V., et al. 2004, MNRAS, 348, 333

Dickinson, M. E., et al. 2002, in HST NICMOS Data Handbook v.5.0, ed. B. Mobasher, Baltimore, STScI

Dobler, G., \& Keeton, C. R. 2005 [arXiv: astro-ph/0502436]

Dunlop, J. S., Taylor, G. L., Hughes, D. H., \& Robson, E. I. 1993, MNRAS, 264, 455

Dye, S., \& Warren, S. J. 2005, ApJ, 623, 31

Elvis, M., Wilkes, B. J., McDowell, J. C., et al. 1994, ApJS, 95, 1

Evans, N. W., \& Witt, H. J. 2003, MNRAS, 345, 1351

Impey, C. D., Falco, E. E., Kochanek, et al. 1998, ApJ, 509, 551

Inoue, K. T., \& Chiba, M. 2005, ApJ, 634, 77

Kawano, Y., Oguri, M., Matsubara, T., \& Ikeuchi, S. 2004, PASJ, 56, 253

Keeton, C. R. 2001, [arXiv: astro-ph/0102340]

Keeton, C. R. 2003, ApJ, 584, 664

Keeton, C. R., Falco, E. E., Impey, C. D., et al. 2000, ApJ, 542, 74

Keeton, C. R., Gaudi, B. S., \& Petters, A. O. 2003, ApJ, 598, 138 (KGP03)

Keeton, C. R., Gaudi, B. S., \& Petters, A. O. 2005, ApJ, 635, 35 (KGP05)

Krist, J. E., \& Hook, R. N. 2003, Tiny Tim Manual, http://www. stsci.edu/software/tinytim/tinytim.html

Kochanek, C. S., \& Dalal, N. 2004, ApJ, 610, 69

Kochanek, C. S., Keeton, C. R., \& Mc Leod, B. A. 2001, ApJ, 547, 50

Macciò, A. V., Moore, B., Stadel, J., \& Diemand, J. 2005 [arXiv: astro-ph/0506125]

Magain, P., Courbin, F., \& Sohy, S. 1998, ApJ, 494, 472

Maller, A. H., Simard, L., Guhathakurta, P., et al. 2000, ApJ, 533, 194

Mao, S., \& Schneider, P. 1998, MNRAS, 295, 587

Mao, S., Jing, Y., Ostriker, J. P., \& Weller, J. 2004, ApJ, 604, L5

Metcalf, R. B. 2005, ApJ, 622, 72

Metcalf, R. B. 2005, ApJ, 629, 673

Metcalf, R. B., \& Madau, P. 2001, ApJ, 563, 9

Metcalf, R. B., \& Zhao, H. 2002, ApJ, 567, 5

Möller, O., Hewett, P., \& Blain, A. W. 2003, MNRAS, 34, 1 
Morgan, N. D., Kochanek, C. S., Pevunova, O., \& Schechter, P. L. Sigurdson, K., \& Kamionkowski, M. 2004, Phys. Rev. Lett., 92, 2005, AJ, 129, 2531

Nenkova, M., Ivezic, Z., \& Elitzur, M. 2002, ApJ, 570, 9

Oguri, M. 2005, MNRAS, 361, L38

Oguri, M., \& Lee, J. 2004, MNRAS, 355, 120 171302

Sluse, D., Surdej, J., Claeskens, J.-F., et al. 2003, A\&A, 406, L43 (Paper I)

Treyer, M., \& Wambsganss, J. 2004, A\&A, 416, 19

Patnaik, A. R., Browne, I. W. A., Walsh, D., Chaffee, F. H., \& Foltz, C. B. 1992, MNRAS, 259, 1

Quadri, R., Möller, O., \& Natarajan, P. 2003, ApJ, 597, 659

Trott, C. M., \& Webster, R. L. 2002, MNRAS, 334, 621

Trott, C. M., \& Melatos, A. 2005, ApJ, 618, 38

Walter, H. G., \& West, R. M. 1986, A\&A, 156, 1

Rozo, E., Zentner, A. R., Bertone, G. F., \& Chen, J. 2005 [arXiv: astro-ph/0506573]

Saha, P., \& Williams, L. L. R. 2004, AJ, 127, 2604

Sánchez, S. F., Jahnke, K., Wisotzki, L., et al. 2004, ApJ, 614, 586

Schechter, P. L., \& Wambsganss, J. 2002, AJ, 580, 685

Schneider, P., \& Weiss, P. 1992, A\&A, 260, 1

Wambsganss, J., \& Paczyński, B. 1991, AJ, 102, 864

Wambsganss, J., Bode, P., \& Ostriker, J. P. 2005, ApJ, 635, L1

Wyithe, J. S. B., Webster, R., Turner, E. L., \& Mortlock, D. J. 2000, MNRAS, 315, 62

Zentner, A. R., \& Bullock, J. S. 2002, Phys. Rev. D, 66, 043003

Zentner, A. R., \& Bullock, J. S. 2003, ApJ, 598, 49 